\title{
Idiopathic Autoimmune Thrombocytopenia Purpura in a 12-year-old boy
}

\author{
ELIZABETH BARROWS, BARBARA KRAJ
}

\begin{abstract}
A 12 year old boy presented to the emergency room with chief complaints of nosebleed and petechiae on lower and upper extremities. The case was adopted for an assignment in a junior level Library Research and Case Studies Presentations course in a distance learning CLS curriculum. The study describes the patient's presentation and differential diagnosis based on laboratory results. The disease background is provided and treatment options are outlined.
\end{abstract}

KEY WORDS: Autoimmune Idiopathic Thrombocytopenic Purpura

Clin Lab Sci 2010;23(4)201

Elizabeth Barrows, MLS(ASCP), Peacehealth Laboratories, Eugene, OR

Barbara Kraj, MS, MLS(ASCP), Medical College of Georgia, School of Allied Health, Augusta, GA

Address for correspondence: Barbara Kraj, MS, MT(ASCP), Medical College of Georgia, School of Allied Health, Department of Biomedical and Radiological Technologies, EC 3336, 987 St. Sebastian Way, Augusta, GA 30912-0800, 706-721-3046, fax: 706-721-7631, bkraj@mcg.edu

Acknowledgements: The first author wishes to thank all at the Medical College of Georgia who supported her throughout her studies as a distance learning student of the Medical Technology Program (presently Clinical Laboratory Science).

\section{CASE INTRODUCTION}

A 12-year-old boy presented to the Emergency Room with a chief complaint of a severe nosebleed and signs of a rash on his arms and legs. Upon examination the boy's rash was diagnosed as petechiae on his extremities.
The patient history provided upon admission revealed that he had recently been ill with flu-like symptoms. There was no family history of bleeding disorders and the boy exhibited no hepatomegaly or splenomegaly. He was admitted to the hospital for monitoring and further testing. The patient's platelet count was observed to be low, while the rest of his complete blood count $(\mathrm{CBC})$ results were found to be within normal limits (Table 1). Coagulation tests were also performed on this patient. Results of these were also found to be within normal limits (Table 1). Antinuclear antibody (ANA) testing also showed negative results, ruling out any bleeding related to Systemic Lupus Erythematosus (SLE).

Table 1. Patient's laboratory results upon admission.

\begin{tabular}{lll}
\hline CBC Results: & & Normal Ranges (children) \\
WBC: & $8.2 \times 10^{3} / \mu \mathrm{l}$ & $5-13 \times 10^{3} / \mu \mathrm{l}$ \\
RBC: & $4.22 \times 10^{6} / \mu \mathrm{l}$ & $4.5-4.8 \times 10^{6} / \mu \mathrm{l}$ \\
HGB: & $12.4 \mathrm{gm} / \mathrm{dL}$ & $11-16 \mathrm{gm} / \mathrm{dL}$ \\
HCT: & $37.0 \%$ & $35-41 \%$ \\
MCV: & $87.6 \mathrm{fL}$ & $82-98 \mathrm{fL}$ \\
MCHC: & $33.5 \mathrm{gm} / \mathrm{dL}$ & $31-37 \mathrm{gm} / \mathrm{dL}$ \\
RDW: & $13.5 \%$ & $11.5-14.2 \%$ \\
PLT: & $2 \times 10^{3} / \mu \mathrm{l}$ & $150-450 \times 10^{3} / \mu \mathrm{l}$ \\
Platelets variably enlarged, with normal granulation \\
Differential Count: & \\
Segs: & $40 \%$ & $29-57 \%(5 \mathrm{y} .0) 40-.68 \%$ (15 y.o.) \\
Bands: & $5 \%$ & not significant below 10 \\
Lymphs: & $45 \%$ & $30-45 \%(7 \mathrm{y} .0) 26-.50 \%$ (15 y.o.) \\
Mono: & $4 \%$ & $4-9 \%$ \\
Eos: & $4 \%$ & $0-3 \%$ \\
Baso: & $2 \%$ & $1-3 \%$ \\
Coagulation Tests: & & \\
Prothrombin Time: & $10.5 \mathrm{sec}$ & $9.0-11.0 \mathrm{sec}$ \\
APTT: & $32.0 \mathrm{sec}$ & $28-36 \mathrm{sec}$ \\
Fibrinogen: & $225 \mathrm{mg} / \mathrm{dL}$ & $200-400 \mathrm{mg} / \mathrm{dL}$ \\
D-Dimer: & $<0.22 \mathrm{ng} / \mathrm{dL}$ & $<0.50 \mathrm{ng} / \mathrm{dL}$ \\
\hline
\end{tabular}




\section{CLINICAL PRACTICE}

Examination by a physician in a suspected case of Idiopathic Thrombocytopenia Purpura (ITP) will often be directed at ruling out possible diseases or conditions which may lead to depleted platelet counts in a patient. In the case of this juvenile patient, the platelet count was repeated and rechecked to confirm the original result of $2000 / \mu \mathrm{l}$. Leukemia and lymphoma were ruled out due to the patient's normal CBC results and normal white blood cell (WBC) differential count. A bone marrow biopsy was not performed in this case, but is a possible option for consideration to definitively rule-out leukemia in a patient. The boy's $\mathrm{CBC}$ also revealed no red cell fragmentation (schistocytes). These test results along with normal coagulations studies rule-out Disseminated Intravascular Coagulation (DIC). A low platelet count would be expected with DIC, while Prothrombin Time (PT) and Activated Partial Thromboplastin Time (APTT) tests could be expected to be increased, D-Dimer results would also be high, and a lower than normal Fibrinogen level would be expected. Since this patient had not been treated with heparin, or exposed to it, heparin contamination or heparin induced thrombocytopenia (HIT) were also ruled out. The normal APTT result indicated that antiphospholipid antibody syndrome was not present, which also made a diagnosis of SLE unlikely. Lupus was not really indicated due to the child's age, but a rule-out was confirmed by the negative ANA test.

\section{DISCUSSION}

Thrombocytopenia is an abnormal decrease in the number of a patient's platelets. A normal CBC count with low platelets and physical signs of petechiae is consistent with an idiopathic thrombocytopenia (of unknown etiology). Petechiae are small spots of hemorrhage that appear on the skin as visual evidence of patient bleeding, and give indication of platelet deficiencies. In suspected ITP, testing is generally aimed at determining the reason or reasons why a patient's platelet levels are low, so are primarily designed to provide a means of diagnosis by exclusion. ${ }^{1}$

Idiopathic Autoimmune Thrombocytopenic Purpura (ITP) is a hemorrhagic autoimmune disease. It is often considered to be a secondary disease, and may be identified with a range of other diseases that show a decrease in the platelet counts of a patient. Generally,
ITP is defined as being of undetermined origin. It is usually chronic in adults, can become a chronic disease in children, and will often present following a viral infection. ${ }^{2}$ A retrospective, population - based study conducted in the UK has revealed several associations of the ITP with other hematological diseases, bleeding disorders, dermatological conditions as well as constitutional symptoms described as malaise, lethargy, and chills. The associations of these comorbidities with ITP were observed before and after the onset of the ITP which the authors of the study had attributed to possible common pathways of disease development. ${ }^{3}$ In ITP, IgG or IgA autoantibodies in the patient's blood bind to antigens on the membranes of circulating platelets, resulting in the destruction of those platelets. Traditional theory suggested that the antibodies originally stimulated by a virus, cross-react with existing platelet epitopes, resulting in ITP. However, according to more recent studies, these autoantibodies, directed against glycoproteins of the integrin family, also prevent formation of new platelets via inhibited megakaryopoiesis or destruction of megakaryocytes within the bone marrow. ${ }^{4}$ Nosebleeds and petechial hemorrhage, as the 12-year-old patient exhibited, are typical symptoms, as well as ecchymoses, and bleeding from the gums and within the gastrointestinal tract. Chronic ITP is diagnosed when thrombocytopenia persists in a patient for greater than 6 months while the acute ITP is characterized by a complete resolution of symptoms within 6 months. Children often have an acute, selflimited form of the disease. ${ }^{5}$ Reportedly, about $44 \%$ of children with chronic ITP will go into remission within 10 years but about $4 \%$ will experience intracranial hemorrhage (ICH) which is the most feared complication of ITP. Bansal et.al. have shown that the prognosis is more favorable in female patients 8 years of age and younger. ${ }^{6}$

The term purpura, or purpuric rash, refers to the petechial symptoms associated with ITP, and is most commonly seen on the lower extremities. It is caused by multiple minute hemorrhages occurring under the patient's skin, giving the appearance of a rash. The significant decrease of circulating platelets in the peripheral blood and autoantibodies bound to the antigens of existing platelets in ITP patients often affects clotting ability, allowing for a higher than 


\section{CLINICAL PRACTICE}

normal incidence of bleeding to occur. While a normal range for platelets may be considered to be 150,000 to 400,000 per cubic millimeter, ITP patients will often be seen with counts less than 100,000 per cubic millimeter. They may also suffer a mild anemia attributed to their bleeding. ${ }^{7}$

\section{TREATMENT}

Platelet counts and bleeding time in ITP patients are closely monitored during treatment. Occult tests are performed on stool, urine, and any vomitus to monitor for internal bleeding. In adults, ITP management is often based on the severity of bleeding in the patient. ${ }^{1}$ Management of ITP in children seems to be an area of disagreement among professionals, with some advocating treatment of patient symptoms, primarily based on low platelet counts, and others advocating a watch-and-wait approach. ${ }^{5}$ Patients are discouraged from participating in contact sports or other activities that may place them at risk for bruising and injury. Active bleeding results in a recommendation of bed-rest to give the body inactive time and to assure protection from possible further accidents or injuries. Elevation of the head is indicated to avoid the possibility of any intracranial bleeds due to intracranial pressure. ${ }^{7}$ Intervention in ITP is often directed at controlling or preventing the mechanisms in the body that lead to platelet destruction.

Two forms of therapy are given intravenously to ITP patients to prevent antibody-coated platelets from being cleared (destroyed) by interfering with the binding of Fc-gamma receptors. These are Intravenous Immunoglobulin (IVIP) therapy and intravenous anti-D rhesus $(\mathrm{Rh})_{0}$ immunoglobulin (IV RhIG). ${ }^{5}$ Concerns have been raised at the use of this therapy with actively bleeding patients, however, and caution is advised in these situations due to the potential for increased bleeding. ${ }^{8}$ Steroid therapy is also a standard treatment, administered as a means of increasing and/or stabilizing platelet counts. Corticosteroids (such as oral prednisone) are administered for several reasons. Their application leads to stabilization of the capillaries, resulting in a reduction of bleeding, and their presence slows the synthesis of antiplatelet antibodies. Corticosteroids also block the destruction of platelets coated with antibody by interfering with this particular action in the reticuloendothelial system. ${ }^{5}$ In patients with acute bleeding conditions, transfusions for platelet replacement therapy may be administered. Splenectomy is a common treatment in chronic refractory cases of adults, but has been a debatable practice in juveniles with ITP. Although there are data connecting splenectomy to high remission rates in juveniles, questions have been raised as to whether remissions are actually due to the procedure or to spontaneous remission, something not uncommon to this age group. ${ }^{9}$ Bansal et.al. reported significant inconsistencies in the percentages of pediatric patients treated by splenectomy in different countries which points to lack of consensus among the practitioners regarding this approach. ${ }^{6}$ Concerns have been raised over possible increases in the infection rates of asplenic children up to 5 years of age. ${ }^{10}$ There are several drugs and other related therapies being utilized in the treatment of refractory ITP in children. Rituximab (monoclonal anti-CD20 antibody) is a newer drug currently being used in ITP treatment in both adults and children. It is a chimeric monoclonal antibody that targets pan-B-cell marker CD-20. This binding stimulates mechanisms leading to cell destruction (apoptosis) and is involved in complement activation and cytotoxicity. ${ }^{11}$ Many of the drugs used to treat adults in chronic cases have undesirable side effects, a concern when providing therapy for children. Options in successful therapies are wanted, to avoid splenectomy in children refractory to standard treatment of ITP. Rituximab seems to have proven efficacy and be related to successful remissions, and is hoped to be a possible therapeutic option for those children refractory to standard ITP treatments. ${ }^{10}$ Recommended drug therapies for children, prior to splenectomy as a treatment, include Axathioprine, Cyclophosphamide, and Cyclic High-Dose Methylprenisolone. ${ }^{12}$ Table 2 outlines one pediatric oncologist's recommended treatment regimen for children with Autoimmune Idiopathic Thrombocytic Purpura. ${ }^{12}$

Future possible treatments have been summarized by Liebman. ${ }^{4}$ They include antibodies against $\mathrm{T}$-cell marker of Tumor Necrosis Factor (TNF) family: CD154 and thrombopoietin (TPO) protein agonists. The latter have been shown to stimulate the development of anti-TPO antibodies and were originally withdrawn from clinical trials and later 


\section{CLINICAL PRACTICE}

reintroduced. A non-protein TPO agonist, Eltrombopag, was shown to increase patients' platelet counts and decrease bleeding incidence while receiving the medication when compared with patients on placebo. ${ }^{13}$

Table 2. Staging of immune thrombocytic purpura (ITP) ${ }^{12}$

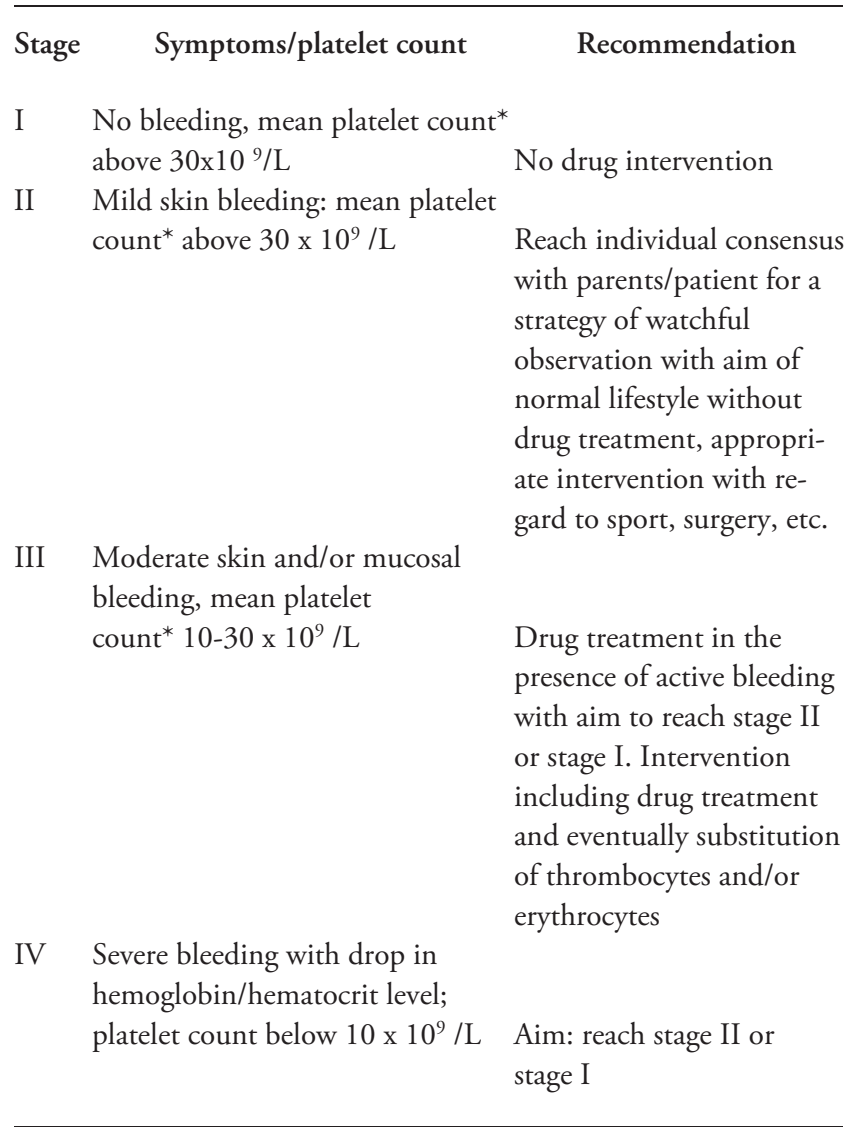

* mean platelet count: mean of 2-3 recent platelet counts within the last 14 days. ${ }^{12}$

\section{CONCLUSION}

ITP is an autoimmune disease affecting platelet counts, with resultant symptoms of bleeding throughout the body. Petechial bleeding, usually on the extremities, epistaxis, gastrointestinal bleeding, bleeding of the gums, and sometimes, anemias related to the bleeding, are typical symptoms leading toward diagnosis. ITP is a diagnosis by exclusion. Treatment generally follows the severity of bleeding in a patient, although there is an argument for treatment of platelet counts in children, with the intent of mediating the severity of the disease. ITP is often chronic in adults and infrequently with children. Children often suffer through an acute phase of the disease, with an eventual recovery, and remain in remission. Corticosteroids, and splenectomy are standard treatment modalites for ITP, although splenectomy is not a recommended choice for children, if possible. Rituximab and Eltrombopag are promising therapeutics, with proponents recommending its use with patient's refractory to standard treatments.

\section{REFERENCES}

1. Cines DB, McMillan R. Management of Adult Idiopathic Thrombocyopenic Purpura. Annu Rev Med 2005;56, 425-42.

2. Omwandho CO, Gruessner SE, Roberts TK, Tinneberg HR. Intravenous immunoglobulin (IVIG): modes of action in the clinical management of recurrent pregnancy loss (RPL) and selected autoimmune disorders. Clin Chem Lab Med 2004;42 (4), 359-70.

3. Freudjo-Tepie MA, Le Roux G, Beach KJ, Bennett D, Robinson NJ. Comorbidities of Idiopathic Thrombocytopenic Purpura: A Population-Based Study. Adv Hematol 2009;2009:963506. Epub 2009 Apr 2.

4. Liebman H. Other Immune Thrombocytopenias. SeminHematol 2007;4 Suppl 5:S24-34

5. Shad AT, Gonzalez CE, Sandler SG. Treatment of Immune Thrombocytopenic Purpura in Children. Pediatric Drugs 2005;7(5), 325-36.

6. Bansal D, Bhamare TA, Trehan A, Ahluvalia J, Varma N, Marwaha RK, Outcome of Chronic Idiopathic Thrombocytopenic Purpura in Children. Pediatr Blood Cancer 2010;54: 403-7.

7. Biderman A, Adler E, Fenton BG, Enright AD, Patwell J, Posner DM, et al. Taber's Cyclopedic Medical Dictionary, F.A. Davis Company 2001;20, 1823-5.

8. Nachman RL. Refractory autoimmune thrombocytopenia: response to antiplatelet therapy. www.thelancet.com 2005;366, 1410.

9. Lillyman J. Management of Childhood Idiopathic Thrombocytopenic Purpura. Br J Haematol 1999;105, 871-5.

10. Pusiol A, Cesaro S, Nocerino A, Picco G, Zanesco L, Bisogno G. Successful treatment with the monoclonal antibody rituximab in two children with refractory autoimmune thrombocytopenia. Eur J Pediatr 2003;163, 305-7.

11. Smith MR. Rituximab (monoclonal anti-CD20 antibody): mechanisms of action and resistance. Oncogene 2003;22, 7359-68.

12. Imbach P. Refractory Idiopathic Immune Thrombocytopenic Purpura in Children. Pediatric Drugs 2003;5 (12), 795-801.

13. Bussel JB, Provan D, Shamsi T, Cheng G, Psaila B, Salama A, et al Effect of eltrombopag on platelet counts and bleeding during treatment of chronic idiopathic thrombocytopenic purpura: a randomized, double-blind, placebo-controlled trial. The Lancet 2009;373(9664):641-8. 